\title{
Stenasellus chapmani $n$. sp. Crustacea Isopoda Asellota des eaux souterraines de Bornéo (Sarawak)
}

\author{
Guy Magniez*
}

\begin{abstract}
SUMMARY
The females of a new species of Stenasellidae have been discovered in Snake Cave (=Gua Siput), in Sarawak, 4th Division, Malaysia (Gunong Mulu National Park, Borneo Island), by Mr. Philip Chapman, of Bristol, U.K., during an expedition of the Royal Geographical Society. The females of another larger species were known previously from several Cambodian caves. This suggests the existence of a general distribution of this family in the underground waters of the Peninsula and of the large Islands of the Indochinese Platform. Nevertheless, we must wait for the discovery of the males of these species to study their relationships, either with the European, or with the African stocks of the family.
\end{abstract}

Stenasellus chapmani n.sp.

Origine du matériel

A l'occasion d'une expédition de la Royal Geographical Society au Sarawak (Ile de Bornéo), des Isopodes aquatiques ont été capturés, en mars 1978, par Monsieur Philip Chapman de Bristol (Grande Bretagne) à l'intérieur de la grotte Snake Cave (=Gua Siput), dans Gunong Mulu National Park, 4th Division (coordonnées du massif de Gunong Mulu: $4^{\circ} 4^{\prime} \mathrm{N}, 114^{\circ} 56^{\prime} \mathrm{E}$ ). Les Asellotes ont été trouvés dans un gour d'eau claire, à fond limoneux, mais à marges de calcite concrétionnée propres. Le gour était alimenté par un minuscule suintement liquide issu d'un joint de stratification de la roche. Température de l'eau: $24^{\circ} \mathrm{C}$. Altitude: 100 mètres (renseignements aimablement communiqués par M. Philip Chapman, que nous remercions vivement pour la confiance qu'il nous témoigne).

\section{Lot capturé}

Trois femelles adultes au repos génital ('oostegites non fonctionnels présents, sous forme de longues lamelles quadrangu-

*Laboratoire de Biologie Animale et Générale, Université de Dijon, 6, Bd. Gabriel, 21100, Dijon, France. 
laires, plus étroites proximalement que distalement et se croisant légèrement au niveau de la ligne médiane sternale). Taille décomptée entre milieu de la marge rostrale du céphalon et pointe caudale du pléotelson: $6,15 \mathrm{~mm}$ (type), 6,10 et 5,90 $\mathrm{mm}$. La description est faite après examen de l'ensemble du lot et la dissection de l'exemplaire $n$. 2. L'échantillon est destiné aux collections du British Museum (Natural History).

\section{Caractères généraux}

Stenasellus chapmani n.sp. est un Sténasellide relativement petit (l'espèce découverte au Cambodge par M.Cl. Boutin, en 1969 dépasse $14 \mathrm{~mm}$ ), mais d'aspect très semblable aux espèces européennes, comme St. virei des Pyréneés.

Céphalon globuleux, plus large que long, à marge rostrale sinueuse (fig. 1). Péréionites à portions pleurales étroites, laissant dépasser latéralement l'expansion externe du coxopodite correspondant (fig. 7). Pléonites I et II libres, bien développés en largeur, avec des pointes pleurales bien marquées (fig. 7). La somme de leurs longueurs équivaut à celle d'un péréionite moyen. Pléotelson subquadrangulaire, plus long que large. Largeurs respectives de la tête, des 7 péréionites, des 2 pléonites

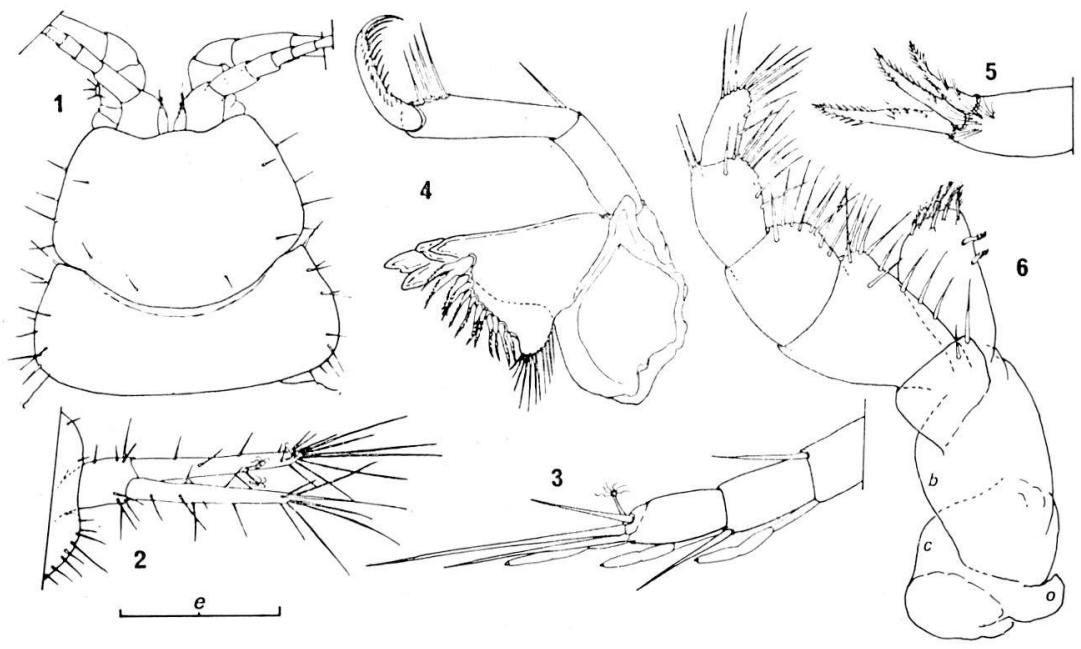

Figures 1-6: 1. Tête et péréionite I, en vue dorsale, de la femelle $\mathrm{n}$. 2, de $6,1 \mathrm{~mm}$, de Stenasellus chapmani n.sp. Les figures n. 2 à 17 se rapportent au même individu; $e=666 \mu$. 2 . Uropode gauche, vue dorsale; $e=666 u$. 3 . Antennule droite, extrémité du fouet; $e=100 \mu$. 4. Mandibule droite; $e=200 \mu$. 5. Maxillule gauche, lobe interne; $e=100 \mu$. 6. Maxillipède droit, face sternale; $b=$ basipodite, $c=$ coxopodite, $o=$ oostégite réduit; $e=200 \mu$. 
libres et du pléotelson: 1,04 - 1,26 - 1,40 - 1,43-1,46 - 1,48 - 1,42 $1,38-1,11-1,13$ et $1,11 \mathrm{~mm}$. Vivants, les Isopodes sont d'une délicate couleur rose (Ph. Chapman, in litt.), vraisemblablement par suite de la présence de pigments hémolymphatiques, comme c'est le cas chez la plupart des Sténasellides.

\section{Description}

Appendices céphaliques: Antennules un peu plus courtes que la hampe de l'antenne; hampe de 4 et fouet de 9 articles, dont le distal très petit et partiellement fusionné avec le pénultième; une lame olfactive hyaline de 75-80 $\mu$ sur chacun des articles $4,5,6,7,8$. Antennes courtes $(3,1 \mathrm{~mm})$, hampe de 6 articles dont le 3ème porte sur sa marge externe une squama très développée et munie de 2-3 épines lisses (fig. 11); fouet avec seulement 30 articles. Paragnathes de grande taille et garnis de fines soies lisses. Mandibules typiques de Sténasellide, à corps court et fortement coudé; palpe triarticulé bien développé (fig. 4); processus inciseur à 4 dents fortement indurées; lacinia mobilis avec 4 dents presque aussi fortes que celles du processus inciseur, suivie d'une rangée de 9 épines dentelées unilatéralement sur

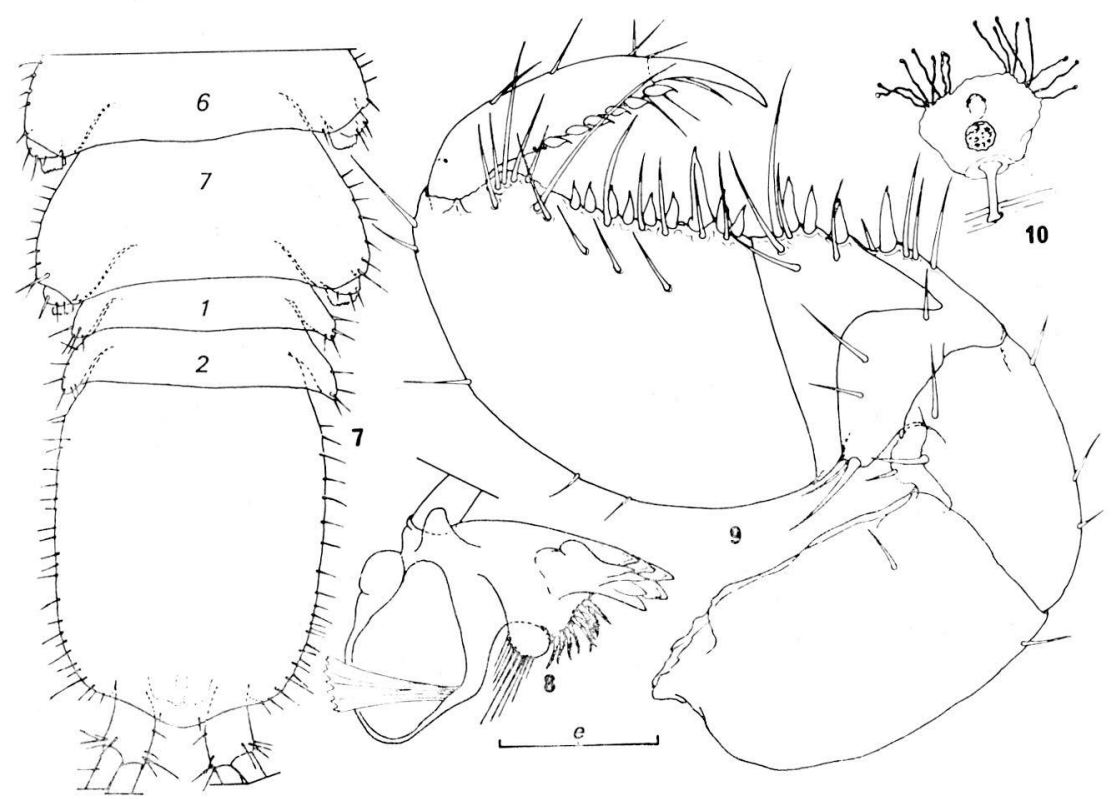

Figures 7-10: 7. Partie postérieure du corps, vue dorsale, 6 et $7=$ péréionites VI et VII, 1 et $2=$ pléonites libres I et II; $\mathrm{e}=666 \mu$. 8. Mandibule gauche, avec lacinia mobilis; $\mathrm{e}=200 \mu$. 9. Péréiopods I droit, face sternale; $\mathrm{e}=200 \mu$. $10 \mathrm{Un}$ des plus gros individus de l'Acinétien pédonculé fixé sur la partie submarginale sternale du propodite du péréiopode I; $\mathrm{e}=40 \mu$. 
la moitié distale de leur marge rostrale (11 épines à la mandibule droite, dont la distale bifide, fig. 4,8); lobe molaire portant 11 - 14 soies simples de longueur variable. Maxillules: lobe externe portant 12 lames denticulées et épines barbelées disposées sur deux rangées distales; leur longueur croit de 50 à $95 \mu$ de l'intérieur vers l'extérieur; lobe interne avec 3 fortes épines rameuses de 40, 50 et $80 \mu$ de long. Maxilles trilobées normales: lobes externe et moyen portant chacun une rangée distale de lames falciforme (respectivement 7 et 6), dentelées sur leur marge interne, dont la longueur décroît respectivement de 125 à $60 \mu$ et de 110 à $60 \mu$, de l'extérieur vers l'intérieur; lobe interne portant 12-13 tiges polymorphes, disposées partiellement sur deux rangées distales et décroissant de 85 à $40 \mu$ vers l'intérieur.

Maxillipédes: Typiques, sans épipodite; rétinacle à 2 crochets; endite portant 15 - 16 fortes tiges ramifiées: 11 - 12 distales (8-9 marginales et 3 submarginales sternales), les 4 dernières, sur-

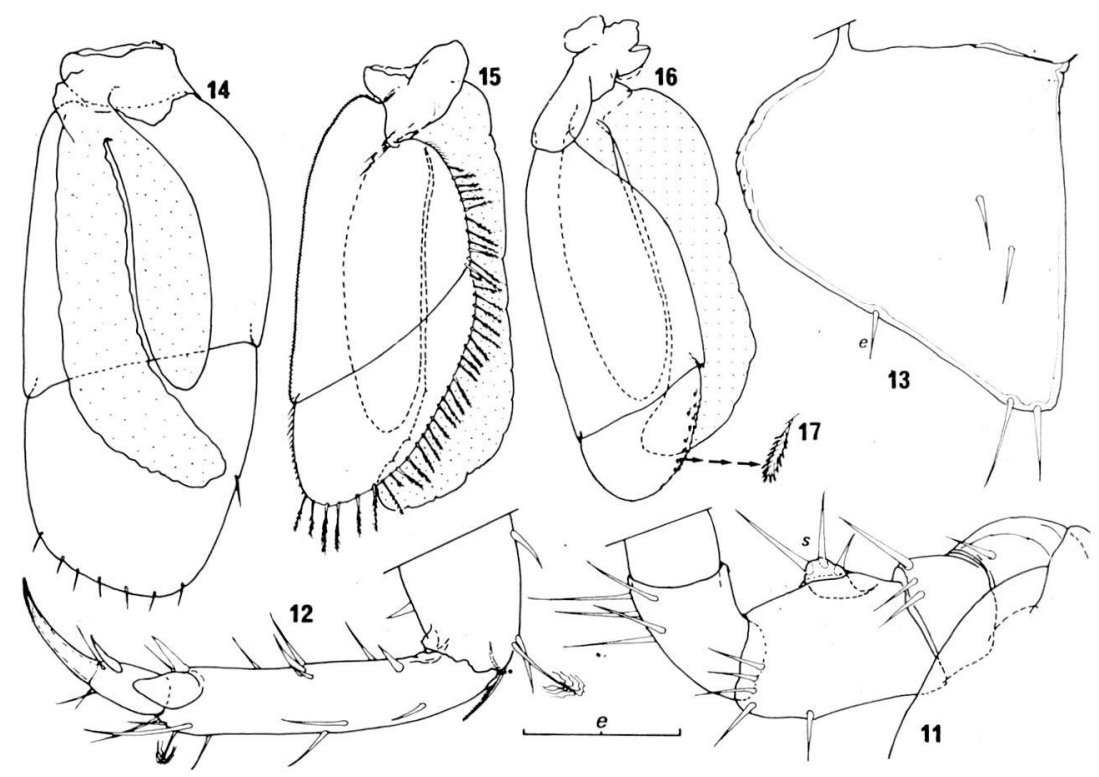

Figures 11-17: 11. Hampe de l'antenne gauche, avec squama (s); e=200 u. 12. Propodite et dactylopodite du péréiopode III droit, face caudale; $e=200 \mu$. 1. Pléopode II droit, face sternale, avec soie exopodiale (e); $e=200 \mu$. 14. Pléopode III droit, face tergale; $\mathrm{e}=400 \mu$. 15. Pléopode IV droit, face sternale; $\mathrm{e}=400 \mu$. 16. Pléopode V droit, face sternale; les endopodites III, IV et V, charnus et respiratoires, sont pointillés; $e=400 \mu .17$. Une des écailles cuticulaires auriculées de la zone marginale interme dẹ l'article distạl de l'exopodite $\mathrm{V} ; \mathrm{e}=40 \mu$. 
tout la proximale, sont plus grandes et forment une rangée tergale; palpe de 5 articles, dont le proximal porte 2 tiges lisses et les autres une dizaine; angle proximal interne du coxopodite avec un petit oostégite charnu (stade de repos génital), (fig. 6).

Péréiopodes I: Conformés en gnathopodes; propodite très renflé et musculeux, à marge sternale à peine concave, munie de deux rangées de tiges ensiformes, entre lesquelles peuvent s'engager les lames tranchantes de la marge sternale du dactylopodite (fig. 9).

Péréiopodes II à VII: Chétotaxie relativement simple; dactylopodites avec une seule épine sternale; marge tergale distale des péréiopodes 2, 3, 4 portant une tige pectinée (fig. 12); longueurs respectives des péréiopodes I à VII: $1,70-2,17-2,16-2,10-2,30$ 2,63 et $2,95 \mathrm{~mm}$; longueurs relatives à P. II: $0,78-0,99-0,96$ $1,06-1,21-1,35$.

Pléopodes II $\$$ : Subtriangulaires et indépendants l'un de l'autre leur taille est assez forte; la soie exopodiale (e) est présente (fig. 13) .

Pléopodes III: Exopodite induré subquadrangulaire, très grand; suture interarticulaire légèrement oblique; quelques courtes soies lisses marginales distales (fig. 14).

Pléopodes IV: Exopodite ovalaire grand, induré, mais mince
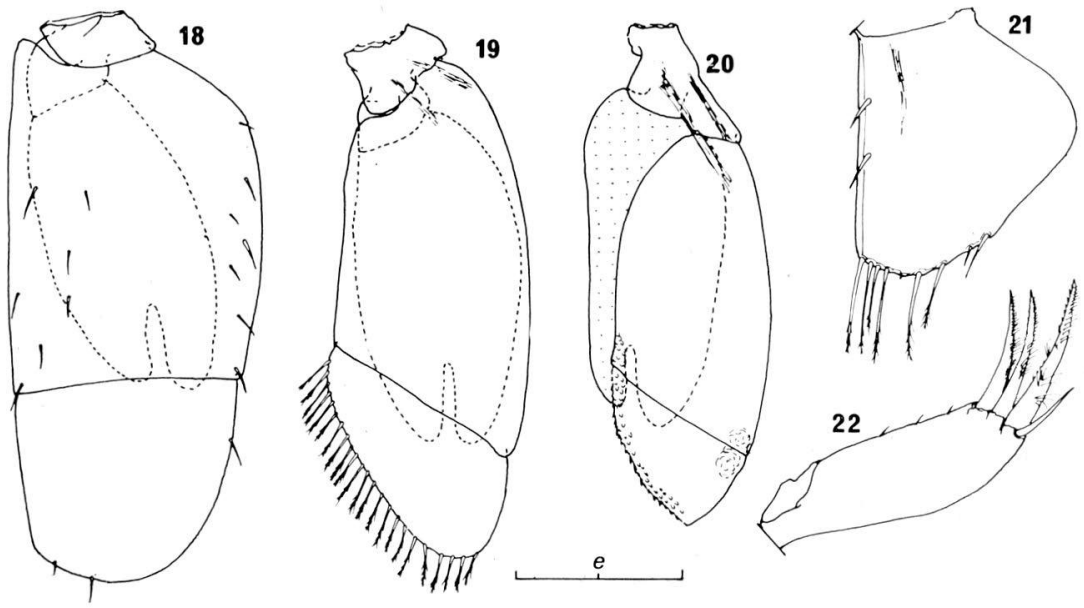

Figures 18-22: 18. Pléopode III gauche de Stenasellus sp. du Cambodge (femelle adulte de $14,5 \mathrm{~mm}$, au repos génital); $\mathrm{e}=1 \mathrm{~mm}$. 19. Pléopode IV gauche de la même; $\mathrm{e}=1 \mathrm{~mm}$. 20. Pléopode V gauche de la même; les endopodites III, IV et V, charnus et respiratoires, sont bifides sur leur quart distal; $e=1 \mathrm{~mm}$. 21. Pléopode II gauche de la même; $e=666 \mu$. 22. Lame interne de la maxillule gauche de la même, avec 3 tiges ramifiées et une soie simple distales; $\mathrm{e}=200 \mu$. 
et hyalin; suture interarticulaire très oblique; marge externe finement ciliée; marge distale et marge interne des deux articles garnie d'une quarantaine de courtes tiges plumeuses (fig. 15).

Pléopodes V: Exopodite subovalaire induré, mince et hyalin; suture interarticulaire à peine oblique; marge distale interne portant de fines écailles épicuticulaires (fig. 16).

Pléopodes III, IV et V: Endopodites charnus, à rôle respiratoire, très développés; complètement bilobés, ils comprennent deux lames disposées côte à côte: la pièce externe, régulièrement ovalaire est plus courte, la pièce interne, plus développée, est bananiforme (figs. 14, 15, 16). D'autres Stenasellidae possèdent ainsi des endopodites III, IV et V bilobés: Stenasellus pardii Lanza de Somalie, genre Balkanostenasellus Cvetkov de Bulgarie et Yougoslavie, Parastenasellus chappuisi (Remy) d'Afrique occidentale, Magniezia studiosorum Sket du Sénégal, mais on ne peut raisonnablement attribuer aucune valeur phylogénétique à un tel caractère, qui apparaît uniquement sur les espèces de petite taille, alors que les espèces de grande taille qui leur sont respectivment apparentées portent des endopodites entiers: Stenasellus pardii $(5 \mathrm{~mm})$ et St. costai Lanza. Messana et Chelazzi (25 mm); Magniezia studiosorum (4-5 mm) et $M$. guinensis (Braga) (18 mm), par exemple. Ainsi, le Stenasellus sp. de grottes du Cambodge, qui dépasse $14 \mathrm{~mm}$, le plus proche géographiquement de St. chapmani n. sp. possède des endopodite III, IV et V seulement bifides distalement (fig. 18, 19, 20).

Uropodes: Très typiques, leur longueur vaut les $9 / 10$ de celle du pléotelson; endopodite légèrement plus long que l'exopodite (fig. 2).

\section{Organisme phorétiques}

Les endopodites des pléopodes III, IV et V, en particulier leur lobe externe, sans doute plus charnu, portent de nombreux Acinétiens sessiles ovoïdes, d'environ $20 \times 30 \mu$. Cependant, un -autre type d'Acinétien pédonculé (fig. 10), très semblable à Choanophrya stenaselli Matjasic, 1963, observé sur les Stenasellus virei hussoni des grottes pyrénéennes, vit fixé en divers points de St. chapmani: angle interne du 3ème article de la hampe de l'antenne, articles 3 et 5 du maxillipède, sternites, à l'abri des oostégites $1,2,3$, sternite thoracique 5 , près des orifices génitaux, mais surtout sur les péréiopodes. Voici la répartition numérique de ces organismes sur les péréiopodes droits de la $q$ n. 2: péréiopode I: basipodite: 2, ischiopodite: 3, méropodite: 4, carpopodite: 8, propodite: 21, dactylopodite: 0: II: 0-2-1-3-0-0; III: 0-1-1-0-0-0; IV: 2-2-0-1-0-0; V: 1-2-1-0-0-0; VI: 0-2-1-1-0-0; VII: 
2-3-1-0-0-0. Sur le propodite I, les Acinétiens s'alignent, en rangées parallèles, au pied des tiges submarginales sternales. Si l'on rappelle que les Sténaselles utilisent principalement leurs péréiopodes I pour capturer et maintenir leurs proies, on constate que les Acinétiens pédonculés sont nombreux là où la nourriture exogène est abondante: ces organişmes phorétiques sont des cleptobiontes, plus que des ectoparasites. Ailleurs que sur le péréiopode I, ils ne subsistent individuellement qu'en des points situés à l'abri des frottements et des nettoyages du Crustacé. Leur abondance laisse présager que, comme chez St. virei, les intermues de St. chapmani n. sp. doivent être de longue durée, malgré la température élevée du milieu.

\section{Remairques systémtiques}

Le mâle de St. chapmani n'étant pas connu, il est hasardeux de chercher à établir les affinités précises de cette forme. L'aspect de l'exopodite du pléopode IV, avec une suture interarticulaire très oblique et surtout une armature de tiges ramifiées s'étendant sur la marge rappelle les espèces du genre Magniezia d'Afrique occidentale, mais il serait prématuré d'y voir un indice de parenté précise et nous attribuerons le Sténasellide de Bornéo au genre Stenasellus, jusqu'à ce qu'une révision générale de ce genre soit possible.

\section{Remarques écologiques et biogêographiques}

Si nous nous référons à l'exemple de Stenasellus virei, la découverte d'un petit nombre d'individus dans un gour d'une grotte est l'indice de l'existence d'un peuplement plus général des eaux souterraines de la région concernée: eaux du massif karstique, mais aussi eaux des nappes phréatiques des bassins hydrographiques adjacents. Des prospections ultérieures seront donc nécessaires pour évaluer l'extension de ces peuplements.

Par ailleurs, l'île de Bornéo constitue une vaste terre émergée $\left(736.000 \mathrm{Km}^{2}\right)$ et il est possible qu'elle recèle plusieurs espèces de Sténasellides. L'existence de ces peuplements insulaires ne pose pas de problèmes biogéographiques insolubles, les îles de Bornéo, Java, Sumatra, faisant partie de la plateforme continentale et ayant été reliées à la péninsule indochinoise lors des régressions quaternaires. On peut même prévoir de futures découvertes de Sténasellides, tant dans les autres grandes îles de la plateforme que dans la péninsule malaise elle-même. 


\section{RESUME'}

Les femelles d'une nouvelle espèce de Stenasellidae ont été découvertes dans la grotte "Snake Cave" (=Gua Sipt), au Sarawak, 4ème division, Fédération de Malaisie (Gunong Mulu National Park, île de Bornéo), par M. Philip Chap. man de Bristol (Grande-Bretagne), à l'occasion d'une expédition de la Royal Geographical Society. Les femelles d'une autre espèce, plus grande, étaient précédemment connues de plusieurs grottes cambodgiennes. Ceci laisse prévoir l'existence d'un peuplement général, par cette famille, des eaux souterraines de la péninsule et des grandes iles de la plateforme indochinoise Cependant, il convient d'attendre la découverte des mâles de ces espèces étudier leurs affinités, soit avec les lignées européennes, soit avec les lignées africaines de la famille.

Manuscrit terminé le 12 décembre 1978.

\section{BIBLIOGRAPHIE}

BOUTIN, Cl. 1971. Observations biospéléologiques en Asie du Sud-Est. Ann. Fac. Sc. Phnom Penh, 4: 168-186.

LANZA, B. 1966. Stenasellus pardii sp. n. della Somalia e note sistematiche su: gli Stenasellinae (Crustacea Isopoda). Monit. zool. ital., 74: 221-256.

MAGNIEZ, G. 1974. Données faunistiques et écologiques sur les Stenasellidae (Crustacea Isopoda Asellota des eaux souterraines). Int. J. Speleol., 6: $1-80$.

MATJASIC, J. 1963. Une nouvelle Choanophrya (Ciliata, Suctoria) sur Stenasellus virei. Ann. spéléol. France, 18: 267-270.

SKET, B. 1969. Eine neue Art der Stenasellinae (Isopoda, Asellota) aus Senegal. Bull. Sci. Univ. Ljubljana, A, 14: 386-387. 